\title{
Oxidation of Adsorbed CO on Pt (111) in CO-saturated Perchloric Acid Aqueous Solutions: Simultaneous In situ Time-Resolved Reflectance Spectroscopy and Second Harmonic Generation Studies
}

Iosif Fromondi and Daniel A. Scherson

\section{SUPPLEMENTARY MATERIAL}

The experimental set up developed for these measurements is shown schematically in Fig. 1. As depicted therein, it involves the use of two focused light sources, a low power laser beam $(633 \mathrm{~nm} \mathrm{CW}$ $\mathrm{HeNe}$, JDS Uniphase, $15 \mathrm{~mW}$ ), and a high power pulsed laser beam (590 nm, 3 ps (HWFH) pulses with a repetition rate of ca. $8 \mathrm{MHz} 30 \mathrm{~mW}$ ) emerging from a Coherent 700 Series Ultrafast Dye Laser with Coherent 7200 Cavity Dumper pumped by a high power laser (Vanguard 2000-HM532 Mode Locked Diode Pumped Solid-State Laser) optimized for $80 \mathrm{MHz}(\leq 12 \mathrm{ps}$ ) pulses at a wavelength of $532 \mathrm{~nm}$. The two laser beams polarized with a broadband polarization rotator (Newport model PR 550) were aimed parallel to one another using a $45^{\circ}$ prism to a single focusing lens with short focal length ( $F=25$ $\mathrm{mm}$ ) to achieve a virtual common focal point on the same facet at an angle of incidence of $45^{\circ}$. The reflected beams were aimed at a focusing lens selected so as to focus the $590 \mathrm{~nm}$ beam onto the photomultiplier (PMT, see below). The transmitted reflectance beam was reflected onto a high reflectance $\mathrm{Al}$ mirror placed at an angle of incidence of $45^{\circ}$ and aimed through yet another focusing lens at the entrance optics of a high-speed amplified silicon detector (ThorLabs PDA155). The reflectance data was collected using one of the four channels of a digital oscilloscope (Tektronix TDS 744A). The polarized $590 \mathrm{~nm}$ laser beam reflecting off the facet was passed first through a polarizer, to control the output polarization and then through a UV filter (Schott UG11) to remove the fundamental frequency, and finally focused onto the entrance slit of a monochromator (Jobin-Yvon H-10) set at a wavelength of $295 \mathrm{~nm}$ (half of the incident wavelength).

The intensity of the p polarized SHG signal, I $(2 \omega)_{\mathrm{pp}}$ at $295 \mathrm{~nm}$, where the subscript pp refers to the $\mathrm{p}$ input and $\mathrm{p}$ output polarization, was measured with a photomultiplier (PMT, Hamamatsu R932) supplied with a nominal voltage of $1.4 \mathrm{kV}$ (Bertran Associates Inc. model 205B-05R) directly attached to the monochromator output. The signal was fed to either of two photon counters (Stanford Research Systems SR400 or Stanford Research Systems SR430). The SR400 was used for scan rates up to $10 \mathrm{~V} / \mathrm{s}$ and the output was recorded and processed using the same 4 channel digital oscilloscope. The SR430 was used for faster scan rates (>10 V/s) the accumulation of the signal being done in independent bins (no of bins and bin size being set independently) when triggered synchronously with other events. The spectroelectrochemical cell used for these in situ dual beam measurements was a 10x10 mm quartz fluorimeter cuvette with five transparent windows. The single-crystal Pt microsphere working electrode (see below) was mounted on the Teflon cap of the cuvette and a 10x10 mm Pt gauze counter electrode was placed at the bottom of the cuvette away from the optical path. A reversible hydrogen electrode (RHE) placed in a different compartment (to avoid CO contamination) and connected to the cell via Teflon tubing inserted into and through the Teflon cap was used as a reference electrode. An EG\&G Model 175 Universal Programmer and Model 173 Potentiostat/Galvanostat was used to scan the input potential to the potentiostat and to trigger initialization of the data acquisition system. The potential and 
currents were collected by the remaining two channels in the oscilloscope. All experiments were performed at room temperature in $0.1 \mathrm{M} \mathrm{HClO}_{4}$ (Ultrex) and/or $0.1 \mathrm{M} \mathrm{H}_{2} \mathrm{SO}_{4}$ (Ultrex) solutions (Barnstead ultrapure water) purged using either UHP Ar (Praxair) or CO (Mathesson Gas purity grade). Single crystal microfacetted microspheres were prepared using Pt wire $50 \mu \mathrm{m}$ in diameter and a hydrogen-air flame. The Pt wire was sealed in soft glass capillary exposing both the microsphere and a small section of the wire to the electrolyte. The size of the (111) microfacets was determined with an optical microscope yielding diameters in the range 20 to $30 \mu \mathrm{m}$.

Fig. 1. Left Panel: Experimental arrangement for in situ simultaneous $\Delta R / R$ and $S H G$ measurements on a Pt(111) facet. Dimensions have been altered for clarity. Right Panels: Plots of averaged, unsmoothed $\Delta \mathrm{R} / \mathrm{R}(\%)$ (upper panel) and $\mathrm{I}(2 \omega)_{\mathrm{pp}}$ (middle panel) vs E, recorded simultaneously for a $\mathrm{Pt}(111)$ facet of a Pt single crystal microsphere in $0.1 \mathrm{M} \mathrm{HClO}_{4}\left(\mathrm{Acq}=400\right.$, black lines) and $0.1 \mathrm{M} \mathrm{H}_{2} \mathrm{SO}_{4}($ Acq $=432$, grey lines) at a scan rate of $2 \mathrm{~V} / \mathrm{s}$. Shown in the lower panel are linear voltammetric scans toward positive potentials of the entire sphere and a section of the connecting wire (left ordinate), as well as the derivatives of the $\Delta \mathrm{R} / \mathrm{R}$ data in the upper panel in this figure (arbitrary units).

Fig. 2. Left Panels. Averaged, smoothed (5-point adjacent average) $\Delta R / R(\%)$ (jagged, thin line, upper panel) and $\mathrm{I}(2 \omega)_{\mathrm{pp}}$ vs $\mathrm{E}$ (thin line, lower panel, right inner ordinate), recorded simultaneously for a $\mathrm{Pt}(111)$ facet of a Pt single crystal microsphere in $\mathrm{CO}$-saturated $0.1 \mathrm{M} \mathrm{HClO}_{4}$ (Acq = 89), while scanning repetitively at a scan rate of $0.1 \mathrm{~V} / \mathrm{s}$ in the potential range $0.05<\mathrm{E}<0.9 \mathrm{~V}$. $\Delta \mathrm{R} / \mathrm{R}$ data were recorded with a home-made low pass RC filter. Similar data collected over the range $0.05<\mathrm{E}<1.1$. V at $50 \mathrm{~V} / \mathrm{s}$ are shown in dotted lines, upper and lower panels, , Acq $=2640(\Delta \mathrm{R} / \mathrm{R})$ and Acq $=2000$ (SHG) outer right ordinate except that a time of $1 \mathrm{~s}$ was allowed at the negative limit to allow for full readsorption of $\mathrm{CO}$. The smooth thick lines are the best fits for $\Delta \mathrm{R} / \mathrm{R}(\%)$ (upper panels, Acq $=6700)$ and $\mathrm{I}(2 \omega)_{\mathrm{pp}}$ (lower panel, Acq $=12600$, left ordinate) vs E recorded in the strict absence of CO in solution at a scan rate of $50 \mathrm{~V} / \mathrm{s}$. Right Panels. Same as left panels for CO-saturated $0.1 \mathrm{M} \mathrm{H}_{2} \mathrm{SO}_{4}$. Data recorded at $0.1 \mathrm{~V} / \mathrm{s}, \mathrm{Acq}=180(\Delta \mathrm{R} / \mathrm{R})$ and $\mathrm{Acq}=180(\mathrm{SHG})$, for $50 \mathrm{~V} / \mathrm{s}$ with $\mathrm{CO}$ in solution, Acq = $1800(\Delta \mathrm{R} / \mathrm{R})$ and $\mathrm{Acq}=2600(\mathrm{SHG})$, for $50 \mathrm{~V} / \mathrm{s}$ in $\mathrm{CO}$ free solutions, $A c q=5050(\Delta \mathrm{R} / \mathrm{R})$ and $\mathrm{Acq}=$ 14900 (SHG). 